\title{
Australian Theses in Astronomy: Abstracts
}

\section{PhD Theses}

\section{Neutral Hydrogen in the Nearby Universe}

\section{David Graeme Barnes}

School of Physics, University of Melbourne, Parkville, Vic. 3052, Australia

Survey observations, using the $21 \mathrm{~cm}$ emission line of $\mathrm{HI}$, and aimed at detecting a population of low surface brightness (LSB), gas-rich galaxies, are presented. In a set of single dish observations directed at the Centaurus and Fornax clusters, and the Eridanus group, one previously uncatalogued, high SB galaxy was detected south of the Fornax cluster. In an interferometric imaging survey of six empty fields, one HI source was detected, the true nature of which remains unknown. The mass density of neutral gas concealed in LSB, HI-rich objects is established to be cosmologically insignificant, consistent with previous results. HI Parkes All Sky Survey (HIPASS) observations of the Centaurus cluster are presented. HI fluxes, masses and velocity widths are accurately measured for 35 cluster galaxies. This sample of galaxies exhibits a normal range of HI properties, and shows no strong evidence for de-clustering of HI selected galaxies in the cluster and near cluster environment.

High resolution $\mathrm{HI}$ imaging observations of a heterogeneous sample of small galaxy groups are analysed. The five galaxy groups studied show a broad range of individual HI properties, e.g. loose groups surrounding LGG 138 and the genuinely compact LGG 455 are identified in HI emission; a massive ring of $\mathrm{HI}$ is discovered encircling two luminous galaxies in the LGG 138 group; a galaxysized mass of HI is found in LGG 455 confined to an extragalactic cloud which exceeds the threshold density for star formation, yet is optically invisible; and the CCG 1 group is established as a chance alignment of Centuarus cluster galaxies. Global results of the study are that: the deficit of HI flux in synthesis imaging data compared to single dish data is put forward as a quantitative measure of the diffuseness of neutral gas in galaxy groups; several groups contain gas-poor galaxies that ordinarily would contain detectable quantities of $\mathrm{HI}$ - this is interpreted as being due to an increased chance of gas-sweeping collisions in the group environment; and some evidence is found to support previous findings that compact groups preferentially occur in loose systems. The general properties of a future HI selected sample of galaxy groups, derived from the HIPASS database, are predicted, and the construction of the sample is briefly described.

VLA observations of $\mathrm{HI}$ in the gravitational lens $2237+0305$ are presented. The galaxy was detected with an HI mass of $5 \cdot 7 \times 10^{9} \mathrm{~h}_{75}^{-2} \mathrm{M}_{\odot}$ and a maximum circular speed $\left(v_{c}\right)$ of $310 \mathrm{~km} \mathrm{~s}^{-1}$. An $r^{\frac{1}{4}}$ bulge is fitted to $v_{c}$, yielding a total bulge mass of $1 \cdot 27 \times 10^{11} \mathrm{~h}_{75}^{-1} \mathrm{M}_{\odot}$. This is found to be identical to the total bulge mass determined by fitting the same bulge profile to the lensing data. The $2237+0305$ system is the only system for which this comparison can and has been made. The line-of-sight velocity dispersion for both fitted bulges is $146 \mathrm{~km} \mathrm{~s}^{-1}$, and is consistent with the observed stellar velocity dispersion. $2237+0305$ is found to be a member of an association of at least five galaxies. The long crossing time of the system ( $\sim 5$ Gyr $)$ implies the group is loose; dynamical mass considerations require a soft, dark potential. Compact groups near lensing galaxies can produce detectable shear in images; however, it is not likely that the loose 2237 group modifies the lensing caused by the central lensing galaxy $2237+0305$.

Algorithms for the processing of HIPASS data measured with the Parkes multibeam receiver are developed and implemented. Bandpass removal, flux calibration, velocity frame conversion and spectral smoothing are applied in real time to 26 spectra having 1024 channels each, every five seconds. Example processing results are presented, along with brief details of alternative processing schemes to suit different objectives. The positional accuracy of the HIPASS data is simulated and measured. For point source detections where the peak flux is greater than $\sim 10$ times the noise level, positions can be determined to within $\sim 2$ arcmin.

In an appendix, a new method for measuring integrated flux in cleaned synthesis images is described. The technique makes no important assumptions about image noise characteristics, and yields integrated fluxes that are self-consistent to better than $\sim 12$ per cent, independent of the region of the dataset selected for flux integration. 


\section{Wide Field Aperture Synthesis Radio Astronomy}

\section{Douglas C.-J. Bock}

School of Physics, University of Sydney, Sydney, NSW 2006, Australia

This thesis is focussed on the Molonglo Observatory Synthesis Telescope (MOST), reporting on two primary areas of investigation. Firstly, it describes the recent upgrade of the MOST to perform an imaging survey of the southern sky. Secondly, it presents a MOST survey of the Vela supernova remnant and follow-up multiwavelength studies.

The MOST Wide Field upgrade is the most significant instrumental upgrade of the telescope since observations began in 1981. It has made possible the nightly observation of fields with area $\sim 5$ square degrees, while retaining the operating frequency of $843 \mathrm{MHz}$ and the pre-existing sensitivity to point sources and extended structure. The MOST will now be used to make a sensitive $\left(\mathrm{rms} \approx 1 \mathrm{mJy} \mathrm{beam}^{-1}\right)$ imaging survey of the sky south of declination $-30^{\circ}$. This survey consists of two components: an extragalactic survey, which will begin in the south polar region, and a Galactic survey of latitudes $|b|<10^{\circ}$. These are expected to take about ten years. The upgrade has necessitated the installation of 352 new preamplifiers and phasing circuits which are controlled by 88 distributed microcontrollers, networked using optic fibre. The thesis documents the upgrade and describes the new systems, including associated testing, installation and commissioning.

The thesis continues by presenting a new highresolution radio continuum survey of the Vela supernova remnant (SNR), made with the MOST before the completion of the Wide Field upgrade. This remnant is the closest and one of the brightest SNRs. The contrast between the structures in the central pulsar-powered nebula and the synchrotron radiation shell allows the remnant to be identified morphologically as a member of the composite class. The data are the first of a composite remnant at spatial scales comparable with those available for the Cygnus Loop and the Crab Nebula, and make possible a comparison of radio, optical and soft X-ray emission from the resolved shell filaments. The survey covers an area of 50 square degrees at a resolution of $43^{\prime \prime} \times 60^{\prime \prime}$, while imaging structures on scales up to $30^{\prime}$. It has been used for comparison with Wide Field observations to evaluate the performance of the upgraded MOST.

The central plerion of the Vela SNR (Vela X) contains a network of complex filamentary structures. The validity of the imaging of these filaments has been confirmed with Very Large Array (VLA) observations at $1.4 \mathrm{GHz}$. Unlike the situation in the Crab Nebula, the filaments are not well correlated with $\mathrm{H} \alpha$ emission. Within a few parsec of the Vela pulsar the emission is much more complex than previously seen: both very sharp edges and more diffuse emission are present. It has been postulated that one of the brightest filaments in Vela $\mathrm{X}$ is associated with the X-ray feature (called a 'jet') which appears to be emanating from the region of the pulsar. However, an analysis of the MOST and VLA data shows that this radio filament has a flat spectral index similar to another more distant filament within the plerion, indicating that it is probably unrelated to the X-ray feature.

\section{Magnetic Fields and Star Formation}

\section{S. E. Byleveld}

Special Research Centre for Theoretical Astrophysics, School of Physics, University of Sydney, NSW 2006, Australia

We present three-dimensional numerical simulations which explore the interplay between magnetic fields and two very different star formation mechanisms. To place this study in its historical context, we firstly present an overview of the main theoretical and observational background of magnetic fields in star forming regions. Following this, the threedimensional, self-gravitating, magnetohydrodynamic numerical code, magnetic-SPHTREE, is introduced and tested. We have developed this code specifically to simulate dynamic star formation mechanisms assuming a 'frozen-in' magnetic field.

A suite of numerical simulations, exploring a particular dynamical magnetically influenced star formation mechanism is presented. In this mechanism, clumps within molecular clouds collide superAlfvénically to produce a shock confined layer. The layer cools radiatively and subsequently fragments. It is this gravitational instability which ultimately results in collapse and protostar formation.

Previous numerical studies have examined the unmagnetised case (e.g. Turner et al. 1995; Whitworth et al. 1995) and have demonstrated, not only the success of this mechanism in forming protostars, but also the propensity for this mechanism to form binary and multiple systems. By incorporating a constant magnetic field into the initial state of the molecular gas system, we investigate the influence of magnetic effects on this mechanism.

Simulations of slow collisions of Jeans stable, self-gravitating, magnetic molecular cloud clumps (with $M \leq 6$ where $M$ is the sonic Mach number) that are not, however, magnetically supported are presented here. In these simulations, high density condensations are observed to form as a result of layer fragmentation. For some of the simulations these condensations are gravitationally bound and thus can be identified as protostars. In other cases, the flux-to-mass ratio is approximately equal to the critical flux-to-mass ratio, indicating that further 
collapse is slow, and occurs on ambipolar diffusion time scales. However, because of the presence of a magnetic field, it is less likely that protostars will form via the clump collision process than previously thought. The conditions under which star formation is likely to occur are restricted by the magnetic field and fewer binary and multiple systems are observed to form.

The development of an additional, original numerical code which combines ambipolar diffusion with smoothed particle magnetohydrodynamics is then discussed in detail. This code was constructed using the magnetic-SPHTREE code as a basis. Armed with a three-dimensional numerical code capable of following the diffusion of the magnetic field in a self-gravitating system, we consider the problem of the collapse of a molecular cloud core. These simulations serve the joint purposes of providing a test of the ambipolar diffusion algorithm, and investigating the role of magnetic field diffusion in core collapse. We identify the quasistatic phase of collapse during which the magnetic field diffuses slowly out of the core. Also, for a simulation with relatively low ion/neutral coupling, both the quasistatic phase and the dynamic collapse phase, in which the density enhancement rate continually increases, are observed. Qualitatively, the nature of the core collapse is as expected from known physical processes, and is comparable to axisymmetric numerical calculations performed by Fiedler and Mouschovias (1993), allowing us to conclude that the ambipolar diffusion code is robust.

Finally the main results are summarised and directions for future work are discussed.

Fiedler, R. A., \& Mouschovias, T. Ch. 1993, ApJ, 415, 680 Turner, J. A., Chapman, S. J., Bhattal, A. S., Disney, M. J., Pongracic, H., \& Whitworth, A. P. 1995, MNRAS, 277, 705

Whitworth, A. P, Chapman, S. J., Bhattal, A.S., Disney, M. J., Pongracic, H., \& Turner, J. A. 1995, MNRAS, 277,727

\section{The Phoenix Multiwavelength Deep Survey}

\section{Andrew Hopkins}

Astrophysics Department, School of Physics, University of Sydney, NSW 2006, Australia

This thesis describes the Phoenix Deep Survey, a multiwavelength survey of a two-degree diameter region. Observations at $1.4 \mathrm{GHz}$ and $843 \mathrm{MHz}$ in the radio continuum, at $\mathrm{R}-, \mathrm{V}-, \mathrm{H}-$ and $\mathrm{K}$-bands in the optical and near-infrared, and of galaxy spectra, are analysed to investigate the nature of the faintest observable radio sources. A catalogue of 1079 radio sources is compiled from these observations, and optical counterparts for 541 of these radio sources are identified. Radio and optical source counts are predicted from models for luminosity functions and are compared with the observational source counts. Radio luminosity evolution at a rate of $Q=3 \cdot 3 \pm 0 \cdot 8$ for starburst galaxies, a rate consistent with other studies, is found to be required to match the observed radio source counts. The bivariate (radio/optical) luminosity function is also modelled and used to predict the bivariate source count distribution, for comparison with observation. The redshift distribution predicted from the bivariate luminosity function is investigated. It is established that, as anticipated from earlier studies, a larger proportion of sub-mJy radio sources are starburst galaxies than at higher flux densities, but there are still significant numbers of AGN sources present. The observational radio luminosity function is investigated, and several interesting sources are examined in more detail.

\section{Obscuration of Quasars by Dust and the Reddening Mechanism in Parkes Quasars Frank Masci}

School of Physics, University of Melbourne, Parkville, Vic. 3052, Australia

Most quasar surveys have been based on criteria which assume strong blue continua or a UV-excess. Any amount of dust along the line-of-sight is expected to drastically extinguish the optical/UV flux leading to a selection bias. Radio surveys however should suffer no bias against extinction by dust. Recently, a large complete sample of radio-selected quasars has become available (the 'Parkes sample'). A majority of these sources exhibits optical-to-nearinfrared continua that are exceedingly 'red', very unlike those of quasars selected optically. The purpose of this thesis, broadly speaking, is to explore the problem of incompleteness in optical quasar surveys due to obscuration by dust, and to interpret the relatively 'red' continua observed in the Parkes quasar sample.

The first part of this thesis explores the observational consequences of an intervening cosmological dust component. A preliminary study explores the effects of different foreground dust distributions (on galaxy-cluster scales to the visible extent of normal galaxies) on obscuration of background sources. Numerical simulations of dusty-galaxies randomly distributed along the line-of-sight with simplified assumptions are then performed, and implications for optical counts of quasars and absorption-line statistics are explored. This foundation is extended by considering the effects of more complicated models of foreground obscuration where the dust content evolves with redshift. The Parkes sample is used to constrain evolutionary and physical properties of dust in intervening systems. The contribution of line-of-sight galactic dust to the reddening observed in this sample is also constrained. 
The second part examines the continuum properties of Parkes quasars in the framework of a number of absorption and emission mechanisms to assess the importance of extinction by dust. Three classes of theories are explored: 'intrinsically red' AGN emission models, dust extinction models, and hostgalaxy light models. Simple models are developed and tested against the available data. Several new correlations between spectral properties are predicted and identified observationally. For the dust model, we explore the effects of dust on soft X-rays and compare our predictions with ROSAT data. Possible physical dust properties are constrained. I then consider the possibility that a 'red' stellar component from the host galaxies contributes to the observed reddening. This contribution is quantified using a novel spectral fitting technique. Finally, an observational study of near-infrared polarisation is presented to distinguish between two models for the reddening: the intrinsic 'synchrotron emission model', and the dust model. Combined with spectral and photometric data, these observations are used to constrain various emission and dust absorption models.

\section{The Red Bright Quasar Survey}

\section{Katrina M. Sealey}

Department of Astrophysics and Optics, University of NSW, NSW 2052, Australia

Quasars are the most luminous objects known in the universe. Study of these objects provides crucial information on the growth of structure and the evolution of the universe. In order to provide new information on the bright optical quasar luminosity function and to find bright quasars that can be used for absorption line studies, we began a new survey for bright quasars, the Red Bright Quasar Survey (RBQS).

The RBQS (an objective prism survey using the UK Schmidt Telescope) plans to cover 2000$3000 \mathrm{deg}^{2}$ of sky to an apparent magnitude of $R=18$, for quasars with redshifts to $z \approx 4$. The survey uses red-sensitive photographic emulsions for the first time in a large area quasar survey. The quasar candidates are selected by ultra-violet excess and using a median template method (implemented for the first time here). In this thesis we present the first results for the RBQS: 220 quasars with measured redshifts found in $210 \mathrm{deg}^{2}$ of sky.

The main conclusions are as follows. The success of our selection techniques was confirmed by an algorithm we designed that generates synthetic quasar spectra. We show the number of quasars in the RBQS sample is consistent with other recent surveys, confirming the incompleteness of the Palomar-Green survey. The evolution of the bright optical quasar luminosity function produced results consistent with no evolution of the most optically luminous quasars between redshifts $z=2-4$. A study was made using high resolution data taken of RBQS2355+0108 (one of the quasars discovered in the survey); measurements have been made that provide upper limits on the deuterium-to-hydrogen ratio D/H. During our analysis we concluded that accumulated forest absorption can give rise to substantial fluctuations in the average Lyman limit from quasar to quasar, causing a potentially large error in $\mathrm{D} / \mathrm{H}$.

\section{MSc Theses}

\section{Optical-Infrared Colours of Dust-obscured QSOs}

\section{Brett Anthony Holman}

School of Physics, University of Melbourne, Parkville, Vic. 3052, Australia

It has been suggested (Webster et al. 1995) that large quantities of dust situated at cosmological distances may affect observations of QSO spectral energy distributions. The possibility that substantial numbers of QSOs are obscured by dust and therefore not detected by traditional, optical survey techniques motivates the current project. A composite QSO SED is constructed using the available literature. This composite is used in conjunction with a simple dust model and $U B V R I J H K$ filters to determine how QSO colours, which are often used to detect QSOs, are affected by differing amounts of dust over the redshift range $0 \cdot 2<z<2 \cdot 8$. It is found that traditional techniques, especially UVX, are insensitive to dust-obscured QSOs, and surveys based on optical-infrared colours are needed instead. A number of alternatives are suggested. Methods for finding normal QSOs, extremely dusty QSOs, and high redshift QSOs are also discussed.

Webster, R. L., Francis, P. J., Peterson, B. A., Drinkwater, M. J., \& Masci, F. J. 1995, Nature, 375, 469

\section{Infrared Spectroscopy of Interstellar $\mathrm{H}_{2} \mathrm{O}$ Ice Analogues}

\section{Marco M. Maldoni}

School of Physics, University College,

University of NSW, Australian Defence Force Academy, Canberra, ACT 2600, Australia

New absorbance spectra of the 3-, 6-, 12- and 44- $\mu \mathrm{m}$ bands of amorphous and crystalline $\mathrm{H}_{2} \mathrm{O}$ ice have been obtained. The first three bands have been acquired using a CsI substrate and the last band using a carbon and a silicate substrate respectively. The spectral appearence of the $3-, 6$ - and $12-\mu \mathrm{m}$ bands at different temperatures as well as the effects, if any, of different substrates on the spectral appearence of 
the $44-\mu \mathrm{m}$ band have been investigated. For each band three sets of measurements have been made. In Series I, the ice film was initially deposited onto the substrate at $10 \mathrm{~K}$ and successive spectra were then obtained at intermediate temperatures as the ice was warmed up to $140 \mathrm{~K}$. The second set, Series II, comprises spectra for ice films directly deposited and measured at temperatures between $10 \mathrm{~K}$ and $140 \mathrm{~K}$. The Series III spectra were obtained for an ice film deposited at $140 \mathrm{~K}$ and then at intermediate temperatures as the ice film was cooled down to $10 \mathrm{~K}$. The 3 - and $12-\mu \mathrm{m}$ band peak wavelength results indicate that the ice undergoes an amorphous-to-crystalline phase transition in the 110-120 K interval. The temperature behaviour of the $6-\mu \mathrm{m}$ band is different from that exhibited by the 3 - and $12-\mu \mathrm{m}$ bands. In the former, no clear phase transition temperature can be discerned from its peak wavelength and bandwidth data. The mid-infrared laboratory results have been used to analyse the spectrum of $\mathrm{OH} 231 \cdot 8+4 \cdot 2$. As a result, we conclude that the identification, made by Soifer et al. (1981), of the $12-\mu \mathrm{m}$ band of $\mathrm{H}_{2} \mathrm{O}$ ice in $\mathrm{OH} 231 \cdot 8+4 \cdot 2$ is probably doubtful. The enstatite $44-\mu \mathrm{m}$ measurements do not show a lowering of the phase transition temperature as reported by Moore et al. (1994). We report no substantial differences between the carbon and enstatite spectra. Comparison of our results with polyethylene substrate data (Smith et al. 1994) also reveals no significant differences in either the bandshape or phase transition temperature.

Moore, M. H., Ferrante, R. F., Hudson, R. L., Nuth, J. A., \& Donn, B. 1994, ApJ, 428, L81

Smith, R. G., Robinson, G., Hyland, A. R., \& Carpenter, G. L. 1994, MNRAS, 271, 481

Soifer, B. T., Willner, S. P., Capps, R. W., \& Rudy, R. J. 1981, ApJ, 250, 631 